can be indistinguishable, and correct identification of the etiology is essential for patient management. PCR is the gold standard for identification of herpes viruses, and PCR can also be used for direct detection of TP from genital lesions. Given the recent global resurgence of syphilis, early diagnosis using PCR is an important tool to supplement serology-based diagnosis of syphilis.

Methods The PlexPCR ${ }^{\circledast}$ VHS assay (SpeeDx) has been developed to detect and differentiate HSV-1, HSV-2, VZV and TP. 211 samples (157 positive and 54 negative) were collected from Melbourne Sexual Health Centre (Victoria, Australia) from January-April 2018. Samples consisted of genital, anal/ rectal, oral and non-genital swabs. The performance of the assay was evaluated at the Victorian Infectious Diseases Reference Laboratory (Victoria, Australia) and compared to reference results from in-house qPCR tests (HSV-1/HSV-2/VZV/ CMV multiplex and TP singleplex). TP detection was also compared to serology results.

Results The sensitivity/specificity of each target compared to in-house qPCR was 100\%/99.4\% for HSV-1, 96.0\%/98.8\% for HSV-2, $100 \% / 100 \%$ for VZV and $100.0 \% / 100.0 \%$ for TP. Analysis of TP PCR results compared to serology are still pending.

Conclusion Molecular diagnosis of genital lesions using PlexPCR VHS allows rapid identification of pathogens with high sensitivity and specificity, enabling appropriate patient management.

Disclosure No significant relationships.

\section{P853 HUMAN FACTORS ENGINEERING TO DRIVE THE DEVELOPMENT OF A NEXT GENERATION COLLI-PEE HOME-BASED FIRST-VOID URINE COLLECTION}

${ }^{1}$ Joke Donné, 'Koen Beyers, ${ }^{2} J u d i t h$ Urlings, ${ }^{1}$ Alejandra Rios-Cortes, ${ }^{1}$ Ronald Van Den Bossche, ${ }^{1}$ Nette Meers, ${ }^{1}$ Quinten Van Avondt, ${ }^{1}$ Tine Provinciael, ${ }^{3}$ Laura Hochstenbach, ${ }^{3}$ Paulette Wauben, ${ }^{3}$ Claire Huijnen, ${ }^{2}$ Bianca Ceccarelli, ${ }^{2}$ Katherine Nelissen, ${ }^{4}$ Vanessa Vankerckhoven*. 'Novosanis N.V., Wijnegem, Belgium; ${ }^{2}$ Happy Aging, Diepenbeek, Belgium; ${ }^{3}$ EIZT, Heerlen, Netherlands; ${ }^{4}$ Novosanis N.V., CEO, Wijnegem, Belgium

\subsection{6/sextrans-2019-sti.895}

Background The first-void urine (first $20 \mathrm{ml}$ of the urine flow) is being used for the detection of sexually transmitted infections (STIs), in particular for the detection of (1) Chlamydia trachomatis, (2) Neisseria gonorrhoeae, (3) Mycoplasma genitalium and (4) Trichomonas. Novosanis' Colli-Pee is an innovative device for standardized, volumetric and hygienic self-collection of first-void urine.

The aim of the study was to design a next generation of the Colli-Pee device optimized in architecture and right materials for postal mailing.

Methods Co-creation sessions by living labs Happy Aging (BE) and EIZT (NL) were organized with 20 volunteers to score four 3D-printed designs. Additionally, at home-based testing allowed assessment of the usability of the new generation Colli-Pee in 120 healthy volunteers; 60 in BE and 60 in NL. Volunteers provided feedback via an online questionnaire on the ease of use, the process of an online urine self-test: from requesting and receiving the Colli-Pee urine collection system to the collection of urine to the returning of the sample by postal mail. The returned samples were checked for leakage and collected volume; as well as return conditions.
Results The prototype with a flexible funnel scored best for time of sampling (8.3), user-friendliness (8.3), and choice of material (8.6). The first results from 38 Belgian participants showed no difference between collected volume for the different pee positions used $(\mathrm{p}=0.996$,Kruskal Wallis Test; mean (SD) in $\mathrm{mL}$ : standing position 10.21 (0.34), forward sitting position 10.00 (0.86), backward sitting position $10.34(0.55)$ ). From 50 Belgian participants, $20 \%$ of the volunteers mentioned that they did not use the instructions to assemble the Colli-Pee and 92\% would recommend the Colli-Pee to others. Furthermore, all returned boxes with a urine sample were intact upon arrival.

Conclusion The next-generation Colli-Pee was well-accepted and offers a solution for home-based collection.

Disclosure No significant relationships.

\section{P854 INTERSPECIES CHIMERAS: A TOOL TO IDENTIFY CHLAMYDIAL VIRULENCE FACTORS}

${ }^{1}$ Mark Fernandez*, ${ }^{2}$ Robert Suchland, ${ }^{2}$ Kevin Hybiske. ' University of Washington, Global Health, Seattle, USA; ${ }^{2}$ University of Washington, Seattle, USA

\subsection{6/sextrans-2019-sti.896}

Background Chlamydia trachomatis is an obligate intracellular bacterium and is the most common notifiable infection in the United States. It spends its entire developmental cycle in a membrane bound cytosolic vacuole termed the inclusion, which protects it from otherwise deleterious host innate immune responses. Interferon gamma (IFN $\gamma$ ) plays a critical role in the clearance of Chlamydia in vitro and in vivo, at least in part by inducing cell-autonomous immunity in infected epithelial cells. Chlamydia muridarum, a rodent pathogen with high genomic synteny to C. trachomatis, is completely susceptible to human cell-autonomous immune responses in vitro. In contrast, C. trachomatis is highly resistant to these IFN $\gamma$ induced responses. In published coinfection experiments, inclusions coinhabited by these species are resistant to recognition by cell-autonomous immunity, suggesting that C. trachomatis has evolved active mechanisms to evade recognition by host cytosolic immune surveillance. These mechanisms are completely unknown.

Methods To identify chlamydial genes that may be involved, we have taken advantage of a previously generated library of interspecies chimeras, each of which has a genome that is predominantly C. trachomatis serovar L2 with discrete regions of C. muridarum genes recombined in (range $=12-113$ recombined genes in each individual chimera). We have used these chimeras in an initial screen looking for ubiquitin recruitment to inclusion membranes - an established marker of cell-autonomous immunity recognition.

Results We have identified four chimeras that are ubiquitinated following IFNy stimulation. These four have zones of recombination overlapping with one another, providing us with 11 candidate genes.

Conclusion This outcome highlights the utility of our chimera library, especially when used to identify genetic factors underlying phenotypes for which C. trachomatis and C. murdarum are disparate. Future characterization of the candidate genes in this screen will identify chlamydial virulence factors that aid in immune evasion of IFN $\gamma$-induced host responses, and may inform design of future vaccines.

Disclosure No significant relationships. 